\title{
Justice involvement patterns, overdose experiences, and naloxone knowledge among men and women in criminal justice diversion addiction treatment
}

\author{
Rachel E. Gicquelais ${ }^{1,2,3,5^{*}}$ (D), Briana Mezuk', Betsy Foxman', Laura Thomas ${ }^{3,4}$ and Amy S. B. Bohnert ${ }^{3,4}$
}

\begin{abstract}
Background: Persons in addiction treatment are likely to experience and/or witness drug overdoses following treatment and thus could benefit from overdose education and naloxone distribution (OEND) programs. Diverting individuals from the criminal justice system to addiction treatment represents one treatment engagement pathway, yet OEND needs among these individuals have not been fully described.

Methods: We characterized justice involvement patterns among 514 people who use opioids (PWUO) participating in a criminal justice diversion addiction treatment program during 2014-2016 using a gender-stratified latent class analysis. We described prevalence and correlates of naloxone knowledge using quasi-Poisson regression models with robust standard errors.

Results: Only $56 \%$ of participants correctly identified naloxone as an opioid overdose treatment despite that $68 \%$ had experienced an overdose and $79 \%$ had witnessed another person overdose. We identified two latent justice involvement classes: low involvement (20.3\% of men, $46.5 \%$ of women), characterized by older age at first arrest, more past-year arrests, and less time incarcerated; and high involvement (79.7\% of men, 53.5\% of women),

characterized by younger age at first arrest and more lifetime arrests and time incarcerated. Justice involvement was not associated with naloxone knowledge. Male participants who had personally overdosed more commonly identified naloxone as an overdose treatment after adjustment for age, race, education level, housing status, heroin use, and injection drug use (prevalence ratio [95\% confidence interval]: men 1.5 [1.1-2.0]).
\end{abstract}

Conclusions: All PWUO in criminal justice diversion programs could benefit from OEND given the high propensity to experience and witness overdoses and low naloxone knowledge across justice involvement backgrounds and genders.

Keywords: Opioids, Overdose, Naloxone, Addiction treatment, Criminal justice system involvement, Latent class analysis

\section{Background}

Mortality from opioid overdose quadrupled from 1999 to 2016 in the United States (US) [1, 2]. To reduce overdose mortality, there is a critical need for overdose education and naloxone distribution (OEND) programs to identify, engage, and train people who use opioids

\footnotetext{
* Correspondence: rgicque1@jhu.edu

${ }^{1}$ Department of Epidemiology, University of Michigan School of Public Health, 1415 Washington Heights, Ann Arbor, Ml 48109, USA

2Department of Epidemiology, Johns Hopkins Bloomberg School of Public Health, 615 N. Wolfe Street, Baltimore, MD 21205, USA

Full list of author information is available at the end of the article
}

(PWUO), as they are both potential overdose victims and bystanders who could respond [3-5]. OEND programs train potential overdose bystanders to identify and respond to an opioid overdose and equip participants with naloxone, an opioid antagonist that reverses the respiratory depression caused by high doses of opioids [6, 7]. Many also provide information about Good Samaritan Laws, which protect persons present at an overdose from legal prosecution for illegal activities discovered when they call 911 [7]. Naloxone distribution to PWUO is cost-effective, especially when combined with

(C) The Author(s). 2019 Open Access This article is distributed under the terms of the Creative Commons Attribution 4.0 International License (http://creativecommons.org/licenses/by/4.0/), which permits unrestricted use, distribution, and 
addiction treatment, and reduces opioid overdose mortality [8-10]. However, as highlighted recently by the US Surgeon General [11], there remains an urgent need to maximize the number of PWUO who are wellpositioned to respond to an overdose or benefit from receipt of naloxone.

OEND programs are increasingly incorporated into addiction treatment services, jails, and prisons given the high prevalence of PWUO in these settings and the elevation in overdose risk following addiction treatment and incarceration [4, 12-14]. Mortality among PWUO is up to 21-fold higher after addiction treatment and up to 129-fold higher in the weeks after incarceration relative to the general population [12, 15]. The elevated risk of overdose during these periods is due to a loss of physiologic tolerance to opioids during periods of incarceration or treatment [16]. A lack of access to medicationassisted treatments and social services (e.g., housing) may further contribute to a return to opioid use and subsequent heightened overdose risk [12]. Pre-release naloxone distribution in corrections institutions has reduced population-level overdose risk [17] and may have benefits for witnessed overdose [18].

The US Surgeon General recently called for an improvement in the pre- and post-release addiction treatment services available to incarcerated PWUO and for a "transition to a less punitive and more health-focused approach" [11]. Herein, we examine one less punitive approach in which the addiction treatment and criminal justice system settings intersect: justice diversion addiction treatment programs, which provide PWUO facing legal prosecution with addiction treatment to reduce sentences or avoid criminal charges [13, 19, 20]. PWUO are referred to justice diversion programs by law enforcement, drug courts, the correctional system, or through parole or probationary boards [19, 21-25]. How best to tailor OEND programs to minimize posttreatment overdose risk among clients of justice diversion addiction treatments has not been thoroughly examined.

This study aims to inform OEND planning using a sample of 514 PWUO in a residential justice diversion addiction treatment program in Michigan. First, we characterize justice involvement preceding diversion (e.g., arrest history, age at first arrest, time incarcerated), history of overdose experiences and witnessed overdose, and naloxone knowledge among PWUO in a justice diversion addiction treatment program. We evaluate these separately by gender to account for potential differences in justice involvement for men and women. Second, we evaluate whether justice involvement history is associated with experiencing or witnessing an overdose, given that prior research has suggested a relationship between criminal justice involvement and overdose risk $[3,5,23$,
26-28]. Finally, we examine the relationship of overdose experiences and justice involvement with naloxone knowledge. Based on prior literature, we hypothesized that we would identify subgroups with higher intensities of involvement that could benefit from targeted OEND due to their low naloxone awareness [23, 27-31]. We also hypothesized that, consistent with prior research, personally experiencing and witnessing an overdose would be associated with higher naloxone knowledge [32-34].

\section{Methods \\ Study description}

The analytic sample was drawn from a previously described study of 817 adult ( $\geq 18$ years) patients receiving treatment for drug or alcohol use disorders in a residential addiction treatment program located in a suburban area of Southeast Michigan during October 2014-January 2016 [35]. This facility served patients living throughout Michigan and received client referrals through contracts with the Michigan Department of Corrections. The typical treatment duration for patients was 60-90 days and patients were separated by gender. Research assistants approached eligible patients (who were aged $\geq 18$ years and able to provide informed consent) about their interest in completing a selfadministered survey to assess eligibility for enrollment in a randomized controlled trial. Interested participants provided informed consent, completed a paper and pencil survey that took approximately $1 \mathrm{~h}$ to finish, and received $\$ 5$ for participating. This analysis uses data from the cross-sectional survey and is not restricted to those who participated in the randomized controlled trial. The University of Michigan Institutional Review Board approved the study protocol.

We restricted the analytic sample to participants whose treatment was prompted by the justice system (excluded $n=40$ participants), had used opioids (heroin or prescription opioids not prescribed by a doctor) in their lifetime (excluded $n=237$ participants), and who had non-missing responses to the measures described below (excluded $n=26$ participants, see Additional file 1: Figure S1). Our analytic sample included 514 PWUO.

\section{Measures \\ Justice involvement}

We quantified participants' cumulative and recent pretreatment justice system interactions using five items: age at first arrest (median 18, range 9-59 years), number of past-year arrests (median 1, range 0-42 arrests), number of lifetime arrests (mode 6-10, categories 1-2, 3-5, $6-10,11-49,50-99$, or 100 or more arrests), number of months during the past year spent in jail or prison (median 5.3, range $0-12$ months), and lifetime number of 
years spent in jail or prison (median 3.5, range 041.3 years). We formed categorical variables using quartile or tertile breaks from distributions in the analytic sample, with modifications when appropriate to enhance interpretability (e.g., juvenile versus adult age at first arrest). Categorical variables included age at first arrest (9-17, 18-20, or 21-59 years), past-year arrests $(0,1-2$, $3-42)$, lifetime arrests $(1-5,6-10, \geq 11)$, past-year time spent in jail or prison $(0-1.9,2-5.9,6-10.9,11-$ 12 months), and total time spent in jail or prison (0-0.9, $1-3.4,3.5-7.4,7.5-41.3$ years).

\section{Personal overdose experiences and witnessed overdose}

Before answering questions, participants read the following definition of an overdose: "The following questions are about experiences with taking too much drugs or medications/pills. This is sometimes called 'poisoning,' 'nodding out,' or an 'overdose' or 'OD."' Participants reported the number of overdoses experienced, timing of their most recent overdose, and substances used during the most recent overdose. Participants then read the definition of a witnessed overdose: "The following questions are about times you have seen someone else taking too much drugs or medications/pills, and/or drinking too much alcohol. This is sometimes called an 'overdose.' When someone has an overdose, they might have blue skin color, convulsions, or difficulty breathing, lose consciousness, collapse, cannot be woken up, or have a heart attack or die." [36] and reported the number of overdoses they witnessed and drugs used by the victim during the most recently witnessed overdose. We formed binary variables for ever experiencing an overdose, experiencing an overdose in the past year, and ever witnessing an overdose. We assessed the number of lifetime personal and witnessed overdoses as three-level categorical variables $(0,1-5$, or $\geq 6)$ and summarized whether the participant's most recent overdose experience and witnessed overdose involved heroin or prescription opioids.

\section{Covariates}

Participants reported whether they had heard of naloxone and identified its purpose as an overdose treatment, drug treatment for opioid dependence, detox, other, or do not know (multiple responses were allowed). For the analysis, we defined naloxone knowledge as having heard of naloxone and correctly identifying its purpose as an overdose treatment. We also examined demographic characteristics, including age $(18-29,30-44,45-$ 67 years), housing (dichotomized into temporary housing [rooming house/hotel, halfway house/group home, inpatient treatment facility/hospital, jail, shelter, or homeless] vs. stable housing [house/apartment or friend/ family member's house]), education (less than high
school/GED or high school/GED or higher), race (black, white, other, or multiple), and ethnicity (Hispanic vs. non-Hispanic). We also summarized substance use characteristics in several time frames, including lifetime and past-year heroin and illicit prescription opioid use (defined as use that was not as prescribed by a doctor). Additionally, we summarized whether participants used heroin for $\geq 7$ consecutive days or injected any substance in the month prior to entering treatment or jail. Finally, we described nonmedical prescription opioid use in the month before entering treatment or jail using four items from the Current Opioid Misuse Measure found to describe nonmedical prescription opioid use in the addiction treatment setting [37, 38]. Specifically, we summarized whether participants reported engaging in any of the following when using prescription opioids: taking prescription opioids belonging to someone else, borrowing prescription opioids from someone else, using more than they were prescribed, or using prescription opioids to treat symptoms other than pain.

\section{Latent class analysis Latent class measurement model}

Latent class analysis (LCA) is a statistical technique used to describe unobserved (i.e., latent) subgroups from patterns of observed variables [39]. It is helpful for identifying clusters (subgroups) of individuals who share patterns of characteristics. Lorvick et al. previously described three classes of justice involvement (low, medium, and high) among women who used drugs in California based on their incarceration history and community corrections involvement [29]. We used LCA to identify subgroups of criminal justice system involvement based on five categorical variables: age at first arrest, past year arrests, lifetime arrests, past year time spent in jail or prison, and total time spent in jail or prison.

We fit LCA models with two to six classes and selected the number of latent classes using a combination of interpretability and model fit indices (Akaike information criterion $[\mathrm{AIC}]$, Bayesian information criterion [BIC], adjusted BIC, and entropy). Smaller values of the AIC and $\mathrm{BIC}$, and larger values of entropy indicate better relative model fit [39]. After selecting the number of classes, we ensured convergence to a globally optimal solution using 1000 random start values. Item response probabilities, which reflect the distribution of each observed justice involvement variable within each justice involvement class, provided the basis for investigator-assigned class labels used to describe each latent class. We completed LCA analyses in SAS version 9.4 using PROC LCA [39].

\section{Justice involvement by gender}

Men and women have different criminal sentencing patterns [40], and the relationship of offenses with drug- 
related mortality differs by gender [31]. Additionally, men and women are treated separately in many residential addiction treatment programs, including the facility where these data were collected. Therefore, we assessed whether the justice involvement measurement model operated similarly in groups defined by gender (men vs. women). We fit the LCA model with and without constraints that required item response probabilities to be equal by gender, testing the null hypothesis of measurement invariance (i.e., that item response patterns were the same for men and women) [39]. We used a likelihood ratio test (LRT) to test for measurement invariance. Rejecting the LRT $(p<0.05)$ implied that the measurement model differed by gender.

\section{Correlates of overdose experience, witnessed overdose, and naloxone knowledge}

We examined whether the prevalence of experiencing or witnessing an overdose differed by justice involvement class. We also assessed whether naloxone knowledge was associated with ever experiencing or witnessing an overdose or with justice involvement. We summarized associations using bivariate and adjusted prevalence ratios from quasi-Poisson regression models with robust standard errors, an approach appropriate for highly prevalent binary outcomes [41, 42]. Adjusted models included sociodemographic characteristics (age, race, housing status, education level) and substance use characteristics (heroin use and injection drug use), as these covariates could be associated with naloxone knowledge or related outcomes and the main exposures for this analysis (overdose, witnessed overdose, and justice involvement) [5, 32, 33, 43, 44]. For regression analyses, we formed a categorical justice involvement variable by assigning participants to their most likely latent justice involvement class (i.e., the modal class assignment approach).

\section{Sensitivity analyses}

We conducted two sensitivity analyses. First, to assess whether the relationships between justice involvement and experiencing an overdose, witnessing an overdose, and naloxone knowledge were robust to the modal class assignment LCA approach, we used the pseudo-class draws approach [45]. We conducted 20 imputations that each assigned participants to a justice involvement class based on LCA posterior probabilities [45]. We repeated quasi-Poisson regressions for each imputed dataset for all associations between justice involvement and overdose outcomes that reached statistical significance using the modal class assignment approach and pooled results using imputation procedures [46]. Second, to examine whether our findings were similar among people who had used opioids recently relative to when they entered treatment, jail, or prison, we re-analyzed the relationships between justice involvement, experiencing an overdose, and witnessing an overdose with naloxone knowledge after restricting the sample to participants who reported using heroin or prescription opioids not prescribed to them in the past year and/or who reported using prescription opioids nonmedically in the month before entering treatment or jail.

\section{Results}

\section{Participant characteristics}

Most participants were white (74.7\%), non-Hispanic (95.3\%), and aged 30-44 years (Table 1). Nearly half were arrested for the first time as juveniles (47.9\%). Most were arrested once or twice in the year before treatment or jail $(41.6 \%)$ while $32.5 \%$ had no arrests. Participants spent a median of 3.5 years in their lifetime and 5.3 months of the past year incarcerated.

Most participants had experienced (68.1\%) and/or witnessed (79.2\%) an overdose, and $42.7 \%$ overdosed in the past year. Only $56.2 \%$ of participants had naloxone knowledge $(62.1 \%$ had heard of naloxone and $90.6 \%$ of those who had heard of it correctly identified it as an overdose treatment).

\section{Gender-stratified justice involvement LCA measurement model}

While the BIC indicated optimal fit for a three-class justice involvement LCA model, the two-class model had higher entropy, larger and more stable classes, and was more interpretable than other models (Table 2). Descriptive analysis suggested that justice involvement characteristics differed by gender (Table 1), and we rejected the null hypothesis of measurement invariance using the LRT and the two class model $\left(\chi^{2}=72.0\right.$, degrees of freedom: $24, p$ value $<0.05$ ), implying that item response probabilities and latent class interpretations differed by gender. Therefore, we used the two-class gender-stratified model for the remainder of analyses.

The gender-stratified model recovered two justice involvement classes for each gender that we termed "high" and "low" involvement (Fig. 1). Men with low justice involvement (20.3\% of men) had an older age at first arrest (median 19, mean 22.2 years), few lifetime arrests (80.6\% had 1-5 arrests), and less incarceration time (lifetime median 0.8 , mean 1.2 years; past year median 4.0 , mean 4.3 months); $72.2 \%$ had $1-2$ arrests in the year before treatment. Men with high justice involvement $(79.7 \%$ of men) were more commonly arrested for the first time as a juvenile (65.3\%), had more past year (median 8 , mean 7.2 months), and lifetime incarceration time (median 6 , mean 8.3 years), and had more lifetime arrests (81.4\% had six or more lifetime arrests). 
Table 1 Sample description of 514 people who use opioids in justice diversion addiction treatment during 2014-2016 by gender

\begin{tabular}{|c|c|c|c|}
\hline & $\begin{array}{l}\text { Total } \\
n(\%)\end{array}$ & $\begin{array}{l}\text { Women } \\
n(\%)\end{array}$ & $\begin{array}{l}\text { Men } \\
n(\%)\end{array}$ \\
\hline Total & $514(100)$ & $151(100)$ & $363(100)$ \\
\hline \multicolumn{4}{|l|}{ Justice involvement ${ }^{\mathrm{a}}$} \\
\hline \multicolumn{4}{|l|}{ Age at 1st arrest (years) } \\
\hline Missing & $2(0.4)$ & $0(0)$ & $2(0.6)$ \\
\hline $9-17$ & $246(47.9)$ & $46(30.5)$ & $200(55.1)$ \\
\hline $18-20$ & $138(26.9)$ & $48(31.8)$ & $90(24.8)$ \\
\hline $21-59$ & $128(24.9)$ & $57(37.8)$ & $71(19.6)$ \\
\hline Median (IQR) & $18(16-20.5)$ & $19(17-22)$ & $17(15-19)$ \\
\hline \multicolumn{4}{|l|}{ Lifetime arrests } \\
\hline Missing & $0(0)$ & $0(0)$ & $0(0)$ \\
\hline $1-5$ & $173(33.7)$ & $61(40.4)$ & $112(30.9)$ \\
\hline $6-10$ & $171(33.3)$ & $49(32.5)$ & $122(33.6)$ \\
\hline 11 or more & $170(32.1)$ & $41(27.2)$ & $129(35.5)$ \\
\hline \multicolumn{4}{|l|}{ Arrests in year before treatment or jail } \\
\hline Missing & $3(0.6)$ & $0(0)$ & $0(0)$ \\
\hline 0 & $167(32.5)$ & $24(15.9)$ & $143(39.4)$ \\
\hline $1-2$ & $214(41.6)$ & $77(51.0)$ & $137(37.7)$ \\
\hline $3-42$ & $130(25.3)$ & $50(33.1)$ & $80(22.0)$ \\
\hline Median (IQR) & $1(0-3)$ & $2(1-3)$ & $1(0-2)$ \\
\hline \multicolumn{4}{|l|}{ Time spent in jail or prison in lifetime (years) } \\
\hline Missing & $7(1.4)$ & $4(2.7)$ & $3(0.8)$ \\
\hline $0-0.9$ & $107(20.8)$ & $66(43.7)$ & $41(11.3)$ \\
\hline $1-3.4$ & $135(26.3)$ & $47(31.1)$ & $88(24.2)$ \\
\hline $3.5-7.4$ & $134(26.1)$ & $23(15.2)$ & $111(30.6)$ \\
\hline $7.5-41.3$ & $131(25.5)$ & $11(7.3)$ & $120(33.1)$ \\
\hline Median (IQR) & $3.5(1-7.5)$ & $1.1(0.3-3)$ & $5(2.3-9.8)$ \\
\hline \multicolumn{4}{|l|}{ Time spent in jail or prison in past year (months) } \\
\hline Missing & $17(3.3)$ & $8(5.3)$ & $9(2.5)$ \\
\hline $0-1.9$ & $119(23.2)$ & $43(28.5)$ & $76(20.9)$ \\
\hline $2-5.9$ & $134(26.1)$ & $59(39.1)$ & $75(20.7)$ \\
\hline $6-10.9$ & $125(24.3)$ & $28(18.5)$ & $97(26.7)$ \\
\hline $11-12$ & $119(23.2)$ & $13(8.6)$ & $106(29.2)$ \\
\hline Median (IQR) & $5.3(2-10)$ & $3.1(1.4-6)$ & $6.5(2.9-12)$ \\
\hline \multicolumn{4}{|l|}{ Overdose experience } \\
\hline Experienced an overdose & $350(68.1)$ & $114(75.5)$ & $236(65.0)$ \\
\hline Most recent overdose involved heroin and/or prescription opioids ${ }^{b}$ & $254(72.6)$ & $87(76.3)$ & $167(70.8)$ \\
\hline Experienced an overdose in the year before treatment & $219(42.7)$ & $83(55.0)$ & $136(37.5)$ \\
\hline \multicolumn{4}{|l|}{ Number of experienced overdoses in lifetime } \\
\hline 0 & $164(31.9)$ & $37(24.5)$ & $127(35.0)$ \\
\hline $1-5$ & $225(43.8)$ & $63(41.7)$ & $162(44.6)$ \\
\hline 6 or more & $125(24.3)$ & $51(33.8)$ & $74(20.4)$ \\
\hline \multicolumn{4}{|l|}{ Witnessed overdose } \\
\hline Witnessed any overdose & $407(79.2)$ & $127(84.1)$ & $280(77.1)$ \\
\hline
\end{tabular}


Table 1 Sample description of 514 people who use opioids in justice diversion addiction treatment during 2014-2016 by gender (Continued)

\begin{tabular}{|c|c|c|c|}
\hline & $\begin{array}{l}\text { Total } \\
n(\%)\end{array}$ & $\begin{array}{l}\text { Women } \\
n(\%)\end{array}$ & $\begin{array}{l}\text { Men } \\
n(\%)\end{array}$ \\
\hline $\begin{array}{l}\text { Most recently witnessed overdose involved } \\
\text { heroin and/or prescription opioids }{ }^{c}\end{array}$ & $339(83.3)$ & $117(92.1)$ & $222(79.3)$ \\
\hline \multicolumn{4}{|l|}{ Number of witnessed overdoses in lifetime } \\
\hline 0 & $107(20.8)$ & $24(15.9)$ & $83(22.9)$ \\
\hline $1-5$ & $269(52.3)$ & $84(55.6)$ & $185(51.0)$ \\
\hline 6 or more & $138(26.9)$ & $43(28.5)$ & $95(26.2)$ \\
\hline \multicolumn{4}{|l|}{ Naloxone knowledge } \\
\hline Heard of naloxone & $319(62.1)$ & $109(66.9)$ & $210(57.9)$ \\
\hline Identified purpose of naloxone ${ }^{d}$ & $289(90.6)$ & $101(92.7)$ & $188(89.5)$ \\
\hline \multicolumn{4}{|l|}{ Demographic and social characteristics } \\
\hline Age (years), Median (IQR) & $34(27-46)$ & $31(26-40)$ & $36(28-48)$ \\
\hline \multicolumn{4}{|l|}{ Race } \\
\hline Black & $83(16.2)$ & $18(11.9)$ & $65(17.9)$ \\
\hline White & $384(74.7)$ & $116(76.8)$ & $268(73.8)$ \\
\hline Other & $13(2.5)$ & $3(2.0)$ & $10(2.8)$ \\
\hline Multiple races & $34(6.6)$ & $14(9.3)$ & $20(5.5)$ \\
\hline Hispanic ethnicity & $24(4.7)$ & $5(3.3)$ & $19(5.2)$ \\
\hline Less than high school education/GED & $83(16.2)$ & $54(14.9)$ & $29(19.2)$ \\
\hline Temporary housing in past 3 months ${ }^{e}$ & $290(56.4)$ & $77(51.0)$ & $213(58.7)$ \\
\hline \multicolumn{4}{|l|}{ Substance use } \\
\hline Lifetime heroin use & $347(67.5)$ & $117(77.5)$ & $230(63.3)$ \\
\hline Heroin use in the past year ${ }^{f}$ & $249(71.9)$ & $93(79.5)$ & $156(67.8)$ \\
\hline $\begin{array}{l}\text { Used heroin } \geq 7 \text { consecutive days during the month }^{\text {before treatment or jailf }}\end{array}$ & $194(55.9)$ & $70(59.8)$ & $124(53.9)$ \\
\hline Lifetime prescription opioid use (not as prescribed a doctor) & $485(94.4)$ & $144(95.4)$ & $341(93.9)$ \\
\hline $\begin{array}{l}\text { Used prescription opioids in the past year (not as prescribed } \\
\text { by a doctor) }\end{array}$ & $271(55.9)$ & $96(66.7)$ & $175(51.3)$ \\
\hline $\begin{array}{l}\text { Took or borrowed prescription opioids belonging to someone } \\
\text { else, took more than prescribed, or used for reasons other } \\
\text { than for pain management }\end{array}$ & $371(72.2)$ & $118(78.1)$ & $253(70.0)$ \\
\hline Injected drugs in the month before entering treatment or jail & $221(43.0)$ & $75(49.7)$ & $146(40.2)$ \\
\hline
\end{tabular}

atatent class analysis allows for missing values in indicators and uses information on available indicators to create classes for participants with missing data. Therefore, totals for justice involvement may not add to the full sample size

${ }^{\mathrm{b}}$ Among those who experienced an overdose. Includes most recent experienced overdose events where the participant reported they used heroin and/or prescription opioids. An additional 7 participants (5 men, 2 women) did not report substances used

'Among those who witnessed an overdose. Includes most recently witnessed overdose events where the participant reported that the victim used heroin and/or prescription opioids. An additional 9 participants ( 6 men, 3 women) did not know or did not report substances used by the victim ${ }^{\mathrm{d}}$ Among those who had heard of naloxone

eIncludes living in a halfway house or group home, inpatient facility, jail, shelter, or homeless

${ }_{\mathrm{f}}^{\mathrm{f}}$ Among those who used heroin in their lifetime. An additional 16 participants ( 10 men, 6 women) reported lifetime heroin use but declined to answer questions about past year heroin use. An additional 5 participants ( 4 men, 1 woman) declined to answer questions about use in the 30 days before entering treatment or jail ${ }^{9}$ Among those who used prescription opioids in their lifetime. An additional 19 participants ( 14 men, 5 women) reported lifetime prescription opioid use but declined to answer questions about past year prescription opioid use

The defining features and item response probabilities among women differed from men. Women with low justice involvement ( $46.5 \%$ of women) were more likely to be arrested at an older age at first arrest $(84.2 \%$ aged $\geq$ 18 years), had few lifetime arrests ( $75.7 \%$ with $1-5$ arrests), and spent less time incarcerated (lifetime median 0.3, mean 0.5 years; past year median 2.6, mean 2.8 months). Women with high justice involvement (53.5\% of women) were younger at their first arrest $(70.3 \%<21$ years), had more lifetime arrests $(50.6 \%$ had $\geq 11$ arrests), and spent more time incarcerated (lifetime median 4.4, mean 2.9 years; past year median 4 , mean 5.4 months). 
Table 2 Fit of latent classes models of justice involvement among a sample of people who use opioids in justice diversion addiction treatment during 2014-2016 $(n=514)$

\begin{tabular}{llllll}
\hline Classes & Log likelihood & AIC & BIC & Adjusted BIC & Entropy \\
\hline 2 & -2897.1 & 595.7 & 701.7 & 622.4 & 0.82 \\
3 & -2852.7 & 532.9 & 694.1 & 573.5 & 0.69 \\
4 & -2841.6 & 536.7 & 753.0 & 591.1 & 0.71 \\
5 & -2822.0 & 523.5 & 795.0 & 591.8 & 0.73 \\
6 & -2809.3 & 524.1 & 850.8 & 606.4 & 0.73 \\
\hline
\end{tabular}

Italic font indicates optimal fit index value of the tested solutions

\section{Correlates of overdose and naloxone knowledge}

We found no differences in prevalence of experiencing or witnessing an overdose by justice involvement in bivariate analyses (Fig. 2). Experiencing an overdose and witnessing an overdose were both positively associated with naloxone knowledge among men and women (Fig. 3). Only $26.0 \%$ of men who had never experienced an overdose were knowledgeable of naloxone, whereas $65.7 \%$ of men with lifetime overdose experience had naloxone knowledge. Among women, 35.1\% of women who had not overdosed had naloxone knowledge vs. 77.3\% who overdosed had naloxone knowledge. High justice involvement was associated with lower naloxone knowledge among men in bivariate analyses.

Men who experienced an overdose in their lifetime were $50 \%$ more likely to have naloxone knowledge than men who had not experienced an overdose after adjustment for age, race, education level, residence in temporary housing, lifetime heroin use, and injection drug use in the 30 days before treatment (PR [95\% CI] 1.5 [1.1-2.0]). Women who experienced an overdose (PR [95\% CI] 1.5 [0.95-2.4], $p=0.08$ ) or witnessed an overdose (PR [95\% CI] 1.4 [0.97-2.1], $p=$ 0.07 ) in their lifetime were marginally more likely to have naloxone knowledge. Naloxone knowledge among men did not differ by justice involvement after adjustment (PR [95\% CI] 0.98 [0.79-1.2]).

\section{Sensitivity analyses}

Assigning justice involvement classes with multiple imputation (i.e., the pseudo-class approach) yielded similar results to modal class assignment. The bivariate association of justice involvement with naloxone knowledge among men was not statistically significant after multiple imputation (PR [95\% CI] 0.79 [0.61-1.0], $p=0.08$ ).

After restricting the analytic sample to participants who reported using heroin or prescription opioids not

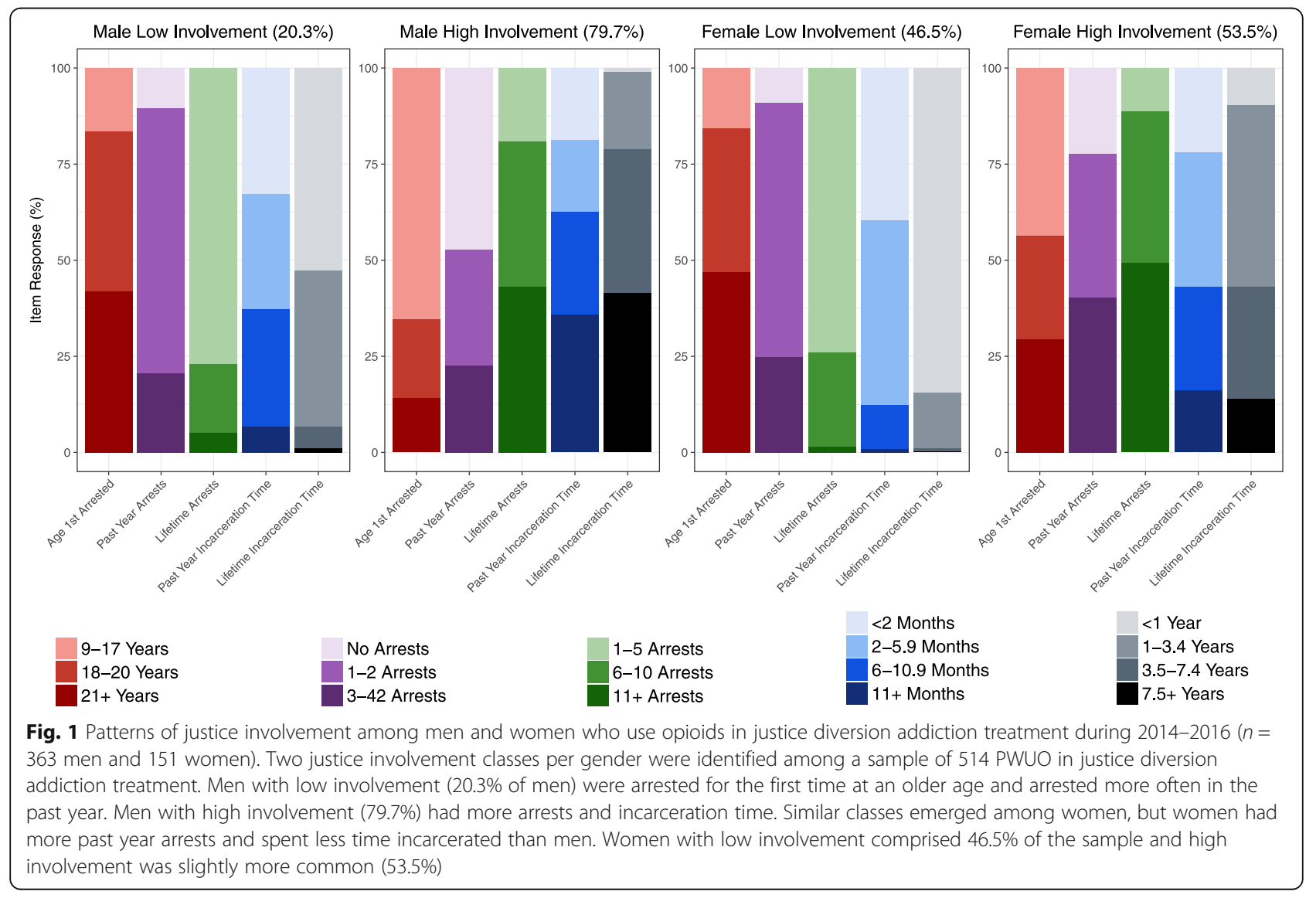




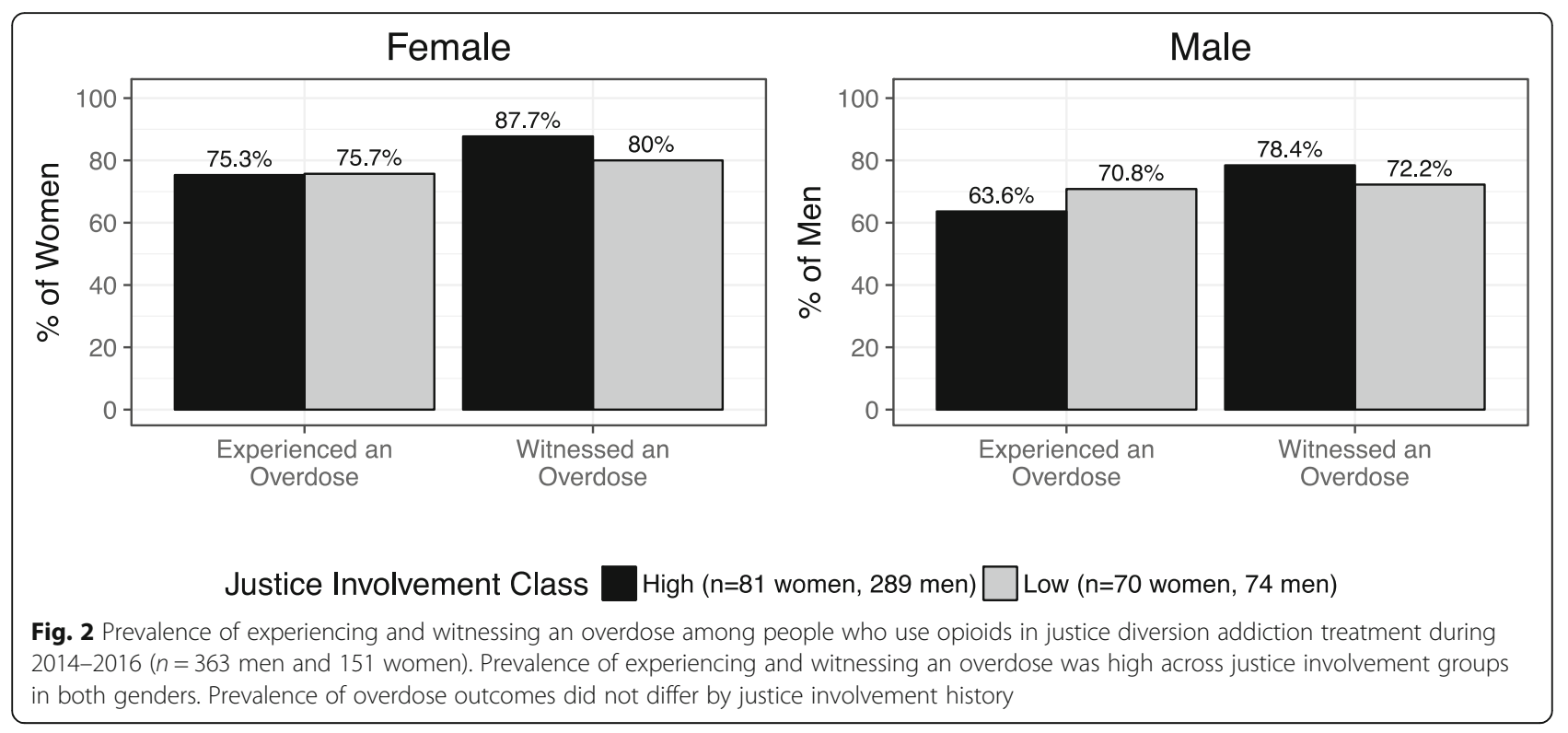

prescribed to them in the past year and/or who reported using prescription opioids nonmedically in the month before entering treatment or jail, 423 participants (288 men, 135 women) remained for analysis. Men who experienced an overdose remained more likely to have naloxone knowledge in adjusted analysis (PR [95\% CI] 1.5 [1.1-2.2], Additional file 1: Table S1). The marginal relationships of experiencing and witnessing an overdose among women were no longer present.

\section{Discussion}

The primary finding of this study was that nearly all PWUO receiving treatment at this residential justice diversion addiction treatment facility during 2014-2016 had experienced and/or witnessed an overdose, but only half had heard of naloxone and correctly identified it as an overdose treatment. Thus, PWUO receiving treatment at this facility are appropriate candidates for OEND given their high likelihood of witnessing or experiencing an overdose after treatment completion [3,

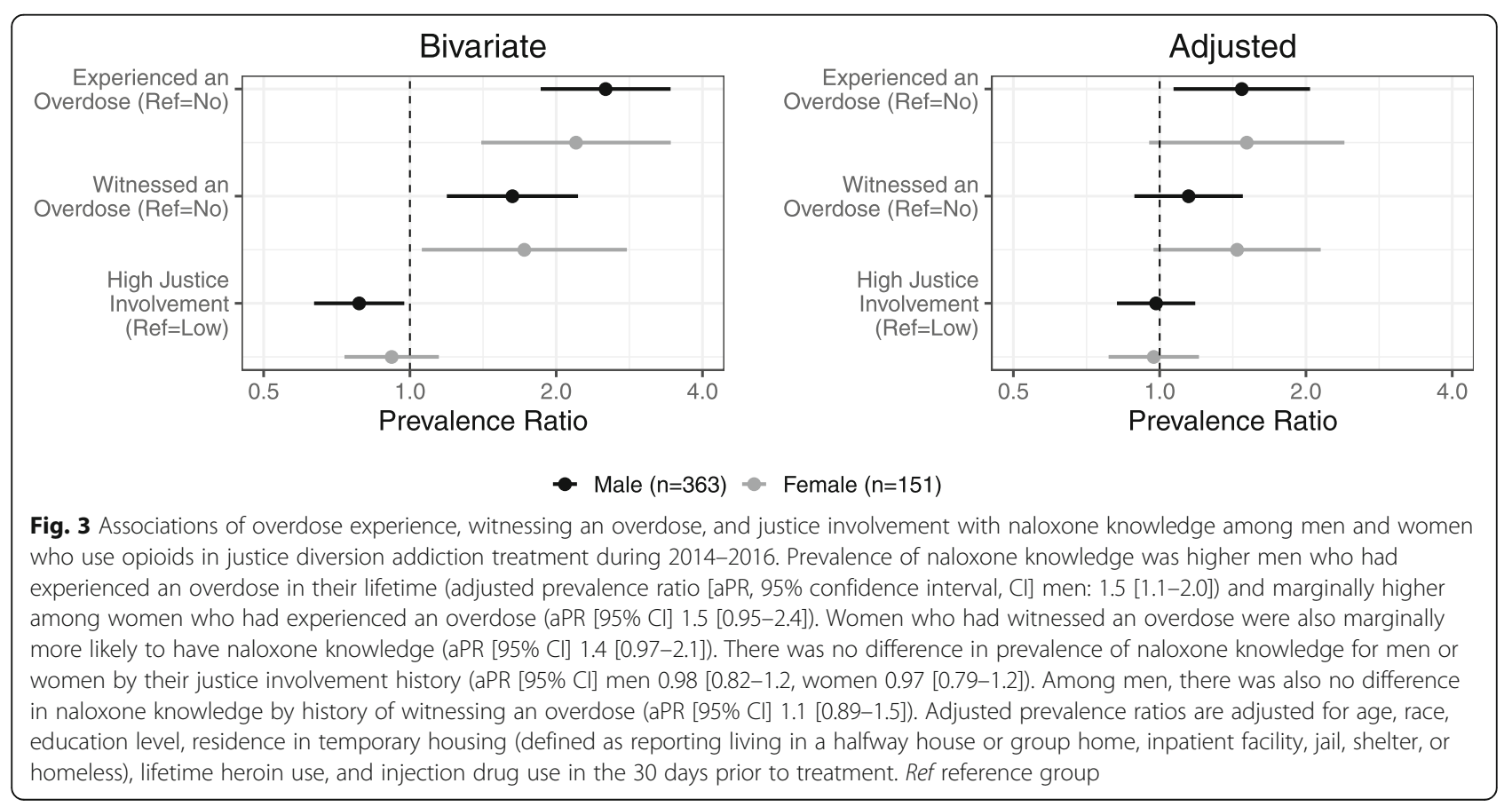


5]. Justice involvement was not associated with naloxone knowledge or with overdose experiences, either personally or as a witness. These findings suggest that clients in justice diversion residential treatment programs in Michigan may be candidates for diversion-based OEND, regardless of their path to treatment. Future work could examine whether OEND scale-up in diversion-based treatment facilities elsewhere should be similarly generalized to all clients or focus on particular PWUO or other subgroups receiving treatment.

In light of continued increases in overdose mortality, the US Surgeon General recently highlighted a need for comprehensive addiction treatment services in jails and prisons and a focus on criminal justice reforms that improve the health of PWUO $[1,2,11]$. We identified two subgroups of PWUO that were defined by simultaneously examining several aspects of their justice involvement history using LCA. These subgroups reflected two pathways that men and women were diverted to treatment in Michigan. Most men (80\%) had long-term justice involvement, whereas few were diverted after many recent arrests, likely to avoid incarceration. Women had had more past year arrests and spent less time incarcerated than men. High and low justice involvement was equally common among women. The patterns of justice involvement in this study reflect both justice involvement patterns among all PWUO and the selection process for diversion programs, such as the one where this study was conducted. For criminal justice reforms to decrease post-incarceration overdose mortality, there is both a need to incorporate OEND into the justice diversion addiction treatment setting and to assess whether current policies divert PWUO at highest risk of returning to opioid use and overdosing after treatment. Whether current diversion program eligibility criteria exclude PWUO at highest risk of post-incarceration overdose given, for example, many recent arrests, is unknown.

The prevalence of experiencing and witnessing an overdose in our study approached the maximum estimates reported in a 2015 systematic review (i.e., $50-96 \%$ of people who use illicit drugs witness an overdose and 17-68\% personally experience an overdose) [5]. The fact that just over half of participants had heard of naloxone and identified it as an overdose treatment, demonstrating lower awareness than has been documented in prior studies [32, 43, 44, 47], highlights the need for the educational component of OEND in this setting. Naloxone knowledge was particularly low among male participants who had never personally experienced an overdose. Results from prior research describing the relationship between personally experiencing an overdose and naloxone knowledge and carrying naloxone have been mixed [32, 48], though one study characterized an association between personal concern for overdose risk and accepting a supply of naloxone in the emergency department [33] and qualitative evidence suggests that PWUO may learn about naloxone from emergency medical providers who respond when they experience an overdose [34]. While we cannot comment on whether these individuals experienced an overdose post-treatment, the fact that they were in addiction treatment, had used opioids, and had no knowledge of naloxone implies that they would benefit from OEND during incarceration or treatment. We found no differences in prevalence of experiencing or witnessing an overdose or in naloxone knowledge by justice involvement, supporting that OEND should be provided to all PWUO in justice diversion addiction treatment.

Our study has several strengths. The LCA approach allowed for a synthesis of several aspects of justice involvement simultaneously. The two groups that emerged were not evident when we examined each justice involvement indicator in isolation. We also had a large sample of PWUO diverted to addiction treatment at several stages post-arrest, which encompassed a variety of potential clients eligible for justice diversion addiction treatment. Another benefit of the large sample size was our ability to stratify our analysis by gender.

Our findings are not without limitation. We studied participants from a single addiction treatment facility located in a suburban area of the mid-Western US and all received treatment because of their criminal justice system involvement. The prevalence of naloxone knowledge, experiencing an overdose, and witnessing an overdose may reflect levels of OEND implementation specific to the Midwest and may not be generalizable outside this region, given that the availability of OEND and other harm reduction services is known to vary geographically $[49,50]$. We were unable to determine when clients were diverted relative to the time they committed the crimes preempting treatment and cannot comment on specific differences between those diverted after arrest, incarceration, or parole/probation. Given the variability in diversion programs, it is difficult to determine whether the patterns of justice involvement observed here would extend to other states [19]. Our ability to evaluate whether our results reflect trends in justice involvement in non-diverted PWUO is limited by the lack of published criteria for diversion program eligibility. This lack of objective criteria further limited our ability to disentangle the sources of gender and other disparities (e.g., by race) in diversion.

Our study relied on self-reported characteristics from the pre-treatment period, potentially introducing recall biases. The cross-sectional design limited our ability to define the temporal sequence of events (e.g., whether individuals experienced or witnessed overdoses after their 
involvement with the criminal justice system). We had no information about participants' access to or experience with syringe services programs or other harm reduction programs or how long they had used opioids, both of which may impact their familiarity with naloxone. Our results may not be generalizable to persons who misused their own prescription opioids as we restricted the analytic sample to participants who selfreported ever using opioids (heroin or prescription opioids not prescribed by a doctor), which may have low sensitivity to misuse of one's own prescribed opioids [51, 52]. Finally, although we had an appropriately high entropy $(>0.8)$ to assign individuals to their most probable justice involvement class, this approach may have underestimated the magnitude of associations between justice involvement with overdose, witnessed overdose, and naloxone knowledge [53-55].

\section{Conclusions}

The low prevalence of naloxone knowledge and high prevalence of experiencing and witnessing an overdose in our sample of PWUO suggests that OEND should be routinely incorporated into justice diversion addiction treatment. Further, OEND should be provided to all clients, regardless of pre-treatment overdose experience or justice involvement characteristics.

\section{Additional file}

Additional file 1: Figure S1. Inclusion Criteria: Selection of Participants for a Study of People who Use Opioids in Justice Diversion Addiction Treatment during 2014-2016. Table S1. Bivariate and Adjusted Logistic Regression Results for Correlates of Naloxone Knowledge among 423 Men and Women in Justice Diversion Addiction Treatment during 20142016 who Used Heroin or Prescription Opioids in the Past Year or who Misused Prescription Opioids in the 30 Days Prior to Jail, Prison, or Attending Treatment. (PDF $188 \mathrm{~kb}$ )

\section{Abbreviations}

AIC: Akaike information criterion; BIC: Bayesian information criterion; Cl: Confidence interval; LCA: Latent class analysis; LRT: Likelihood ratio test; OEND: Overdose education and naloxone distribution; PR: Prevalence ratio; PWUO: People who use opioids; US: United States

\section{Acknowledgements}

We thank Emily Yeagley for providing project oversight. We also thank the participants and staff at the addiction treatment facility who facilitated this research.

\section{Funding}

This work was supported by the National Institutes of Health (R34 DA035331 and T32 Al102623) and the University of Michigan Integrated Training in Microbial Systems (Burroughs Wellcome Trust).

\section{Availability of data and materials}

The datasets generated and/or analyzed for the current study are available from the corresponding author on reasonable request.

\section{Authors' contributions}

ASBB conceptualized the study and obtained funding. LT and ASBB collected data. RG conducted analyses, obtained additional funding, and drafted the manuscript. All authors conceptualized the analysis design, and read, edited, and approved the final manuscript.

Ethics approval and consent to participate

The University of Michigan Institutional Review Board approved the study protocol. Participants provided written informed consent.

\section{Consent for publication}

Consent for publication was obtained.

\section{Competing interests}

The authors declare that they have no competing interests.

\section{Publisher's Note}

Springer Nature remains neutral with regard to jurisdictional claims in published maps and institutional affiliations.

\section{Author details}

${ }^{1}$ Department of Epidemiology, University of Michigan School of Public Health, 1415 Washington Heights, Ann Arbor, MI 48109, USA. ${ }^{2}$ Department of Epidemiology, Johns Hopkins Bloomberg School of Public Health, $615 \mathrm{~N}$. Wolfe Street, Baltimore, MD 21205, USA. ${ }^{3}$ Department of Psychiatry, University of Michigan, 2800 Plymouth Road, Ann Arbor, Ml 48109, USA. ${ }^{4}$ Veterans Affairs Center for Clinical Management Research, 2215 Fuller Road, Ann Arbor, MI 48105, USA. ${ }^{5}$ Current Address: Johns Hopkins Bloomberg School of Public Health, 615 N. Wolfe St, E7133A, Baltimore, MD 21205, USA.

Received: 16 May 2019 Accepted: 5 July 2019

Published online: 16 July 2019

\section{References}

1. Rudd RA, Aleshire N, Zibbell JE, Gladden RM. Increases in drug and opioid overdose deaths_-United States, 2000-2014. MMWR Morb Mortal Wkly Rep. 2016;64(50-51):1378-82.

2. Seth P. Scholl L, Rudd RA, Bacon S. Overdose deaths involving opioids, cocaine, and psychostimulants-United States, 2015-2016. MMWR Morb Mortal Wkly Rep. 2018;67 [cited 2018 May 4]. Available from: https://www. cdc.gov/mmwr/volumes/67/wr/mm6712a1.htm

3. Bohnert ASB, Tracy M, Galea S. Characteristics of drug users who witness many overdoses: implications for overdose prevention. Drug Alcohol Depend. 2012;120(1-3):168-73.

4. Lagisetty P, Bohnert A, Fendrick A. Meeting the opioid challenge: getting naloxone to those who need it most. Health Affairs Blog. 2018; [cited 2018 May 22]. Available from: https://www.healthaffairs.org/do/10.1377/hblog201 80510.164285/full/

5. Martins SS, Sampson L, Cerdá M, Galea S. Worldwide prevalence and trends in unintentional drug overdose: a systematic review of the literature. Am J Public Health. 2015:105(11):2373.

6. Behar E, Santos G-M, Wheeler E, Rowe C, Coffin PO. Brief overdose education is sufficient for naloxone distribution to opioid users. Drug Alcohol Depend. 2015;148:209-12.

7. Mueller SR, Walley AY, Calcaterra SL, Glanz JM, Binswanger IA. A review of opioid overdose prevention and naloxone prescribing: implications for translating community programming into clinical practice. Subst Abuse. 2015:36(2):240-53

8. McDonald R, Strang J. Are take-home naloxone programmes effective? Systematic review utilizing application of the Bradford Hill criteria. Addict Abingdon Engl. 2016;111(7):1177-87.

9. Coffin PO, Sullivan SD. Cost-effectiveness of distributing naloxone to heroin users for lay overdose reversal. Ann Intern Med. 2013;158(1):1-9.

10. Uyei J, Fiellin DA, Buchelli M, Rodriguez-Santana R, Braithwaite RS. Effects of naloxone distribution alone or in combination with addiction treatment with or without pre-exposure prophylaxis for HIV prevention in people who inject drugs: a cost-effectiveness modelling study. Lancet Public Health. 2017;2(3):e133-40

11. HHS (US Department of Health and Human Services Office of the Surgeon General). Facing addiction in America: the surgeon General's spotlight on opioids. Washington, DC: HHS; 2018.

12. Binswanger IA, Stern MF, Deyo RA, Heagerty PJ, Cheadle A, Elmore JG, et al. Release from prison - a high risk of death for former inmates. N Engl J Med. 2007;356(2):157-65. 
13. Brinkley-Rubinstein L, Cloud DH, Davis C, Zaller N, Delany-Brumsey A, Pope $L_{\text {, et }}$ al. Addressing excess risk of overdose among recently incarcerated people in the USA: harm reduction interventions in correctional settings. Int J Prison Health. 2017:13(1):25-31.

14. Merrall ELC, Kariminia A, Binswanger IA, Hobbs MS, Farrell M, Marsden J, et al. Meta-analysis of drug-related deaths soon after release from prison. Addict Abingdon Engl. 2010;105(9):1545-54.

15. Davoli M, Bargagli AM, Perucci CA, Schifano P, Belleudi V, Hickman M, et al. Risk of fatal overdose during and after specialist drug treatment: the VEdeTTE study, a national multi-site prospective cohort study. Addict Abingdon Engl. 2007;102(12):1954-9.

16. Strang J, McCambridge J, Best D, Beswick T, Bearn J, Rees $S$, et al. Loss of tolerance and overdose mortality after inpatient opiate detoxification: follow up study. BMJ. 2003;326(7396):959-60.

17. Bird SM, McAuley A, Perry S, Hunter C. Effectiveness of Scotland's National Naloxone Programme for reducing opioid-related deaths: a before (2006-10) versus after (2011-13) comparison. Addict Abingdon Engl. 2016;111(5):88391.

18. Parmar MKB, Strang J, Choo L, Meade AM, Bird SM. Randomized controlled pilot trial of naloxone-on-release to prevent post-prison opioid overdose deaths. Addict Abingdon Engl. 2017;112(3):502-15.

19. Brinkley-Rubinstein L, Zaller N, Martino S, Cloud DH, McCauley E, Heise A, Seal D. Criminal justice continuum for opioid users at risk of overdose. Addict Behav. 2018;86:104-10.

20. Horton M, McDonald R, Green TC, Nielsen S, Strang J, Degenhardt L, et al. A mapping review of take-home naloxone for people released from correctional settings. Int J Drug Policy. 2017;46:7-16.

21. Abreu D, Parker TW, Noether CD, Steadman HJ, Case B. Revising the paradigm for jail diversion for people with mental and substance use disorders: intercept 0. Behav Sci Law. 2017;35(5-6):380-95.

22. Belenko S, Logan TK. Delivering more effective treatment to adolescents: improving the juvenile drug court model. J Subst Abus Treat. 2003;25(3): $189-211$.

23. Hser Y-I, Evans E, Grella C, Ling W, Anglin D. Long-term course of opioid addiction. Harv Rev Psychiatry. 2015;23(2):76-89.

24. Johnston EL. Retributive justifications for jail diversion of individuals with mental disorder. Behav Sci Law. 2017;35(5-6):396-407.

25. Sirotich $\mathrm{F}$. The criminal justice outcomes of jail diversion programs for persons with mental illness: a review of the evidence. J Am Acad Psychiatry Law Online. 2009:37(4):461-72.

26. Bohnert ASB, Tracy M, Galea S. Circumstances and witness characteristics associated with overdose fatality. Ann Emerg Med. 2009;54(4):618-24.

27. Hakansson A, Schlyter F, Berglund M. Factors associated with history of nonfatal overdose among opioid users in the Swedish criminal justice system. Drug Alcohol Depend. 2008;94(1):48-55.

28. Wagner KD, Liu L, Davidson PJ, Cuevas-Mota J, Armenta RF, Garfein RS Association between non-fatal opioid overdose and encounters with healthcare and criminal justice systems: identifying opportunities for intervention. Drug Alcohol Depend. 2015;153:215-20.

29. Lorvick J, Comfort M, Kral AH, Lambdin BH. Exploring lifetime accumulation of criminal justice involvement and associated health and social outcomes in a community-based sample of women who use drugs. J Urban Health Bull N Y Acad Med. 2018;95(4):584-93.

30. Barocas JA, Baker L, Hull SJ, Stokes S, Westergaard RP. High uptake of naloxone-based overdose prevention training among previously incarcerated syringe-exchange program participants. Drug Alcohol Depend. 2015;154:283-6.

31. Kariminia A, Law MG, Butler TG, Corben SP, Levy MH, Kaldor JM, et al. Factors associated with mortality in a cohort of Australian prisoners. Eur J Epidemiol. 2007;22(7):417-28.

32. Nolan S, Buxton J, Dobrer S, Dong H, Hayashi K, Milloy MJ, et al. Awareness, possession, and use of take-home naloxone among illicit drug users, Vancouver, British Columbia, 2014-2015. Public Health Rep Wash DC 1974 2017:132(5):563-9.

33. Kestler A, Buxton J, Meckling G, Giesler A, Lee M, Fuller K, et al. Factors associated with participation in an emergency department-based takehome naloxone program for at-risk opioid users. Ann Emerg Med. 2017; 69(3):340-6.

34. Heavey SC, Chang Y-P, Vest BM, Collins RL, Wieczorek W, Homish GG. 'I have it just in case' - naloxone access and changes in opioid use behaviours. Int J Drug Policy. 2018;51:27-35.
35. Fernandez AC, Bush C, Bonar EE, Blow FC, Walton MA, Bohnert ASB. Alcohol and drug overdose and the influence of pain conditions in an addiction treatment sample. J Addict Med. 2019;13(1):61-8.

36. Tracy M, Piper TM, Ompad D, Bucciarelli A, Coffin PO, Vlahov D, et al. Circumstances of witnessed drug overdose in new York City: implications for intervention. Drug Alcohol Depend. 2005;79(2):181-90.

37. Butler SF, Budman SH, Fernandez KC, Houle B, Benoit C, Katz N, et al. Development and validation of the current opioid misuse measure. Pain. 2007 Jul;130(1-2):144-56.

38. Ashrafioun L, Bohnert ASB, Jannausch M, Ilgen MA. Evaluation of the current opioid misuse measure among substance use disorder treatment patients. J Subst Abus Treat. 2015;55:15-20.

39. Lanza ST, Collins LM, Lemmon DR, Schafer JL. PROC LCA: a SAS procedure for latent class analysis. Struct Equ Model Multidiscip J. 2007;14(4):671-94.

40. Doerner JK, Demuth S. The independent and joint effects of race/ethnicity, gender, and age on sentencing outcomes in U.S. Federal Courts. Justice Q. 2010;27(1):1-27.

41. Zou G. A modified Poisson regression approach to prospective studies with binary data. Am J Epidemiol. 2004:159(7):702-6.

42. Richardson DB, Kinlaw AC, MacLehose RF, Cole SR. Standardized binomial models for risk or prevalence ratios and differences. Int J Epidemiol. 2015; 44(5):1660-72.

43. Dietze PM, Stare M, Cogger S, Nambiar D, Olsen A, Burns L, et al. Knowledge of naloxone and take-home naloxone programs among a sample of people who inject drugs in Australia: variations across capital cities. Drug Alcohol Rev. 2018;37(4):457-63.

44. Tobin K, Clyde C, Davey-Rothwell M, Latkin C. Awareness and access to naloxone necessary but not sufficient: examining gaps in the naloxone cascade. Int J Drug Policy. 2018;59:94-7.

45. Bandeen-Roche K, Miglioretti DL, Zeger SL, Rathouz PJ. Latent variable regression for multiple discrete outcomes. J Am Stat Assoc. 1997;92(440): 1375-86.

46. Rubin DB. Multiple imputation for nonresponse in surveys. New York: Wiley; 1987. p. 326.

47. Kirane $\mathrm{H}$, Ketteringham M, Bereket S, Dima R, Basta A, Mendoza S, et al. Awareness and attitudes toward intranasal naloxone rescue for opioid overdose prevention. J Subst Abus Treat. 2016;69:44-9.

48. Tobin KE, Latkin CA. The relationship between depressive symptoms and nonfatal overdose among a sample of drug users in Baltimore, Maryland. J Urban Health Bull N Y Acad Med. 2003;80(2):220-9.

49. Des Jarlais DC, Nugent A, Solberg A, Feelemyer J, Mermin J, Holtzman D. Syringe service programs for persons who inject drugs in urban, suburban, and rural areas-United States, 2013. MMWR Morb Mortal Wkly Rep. 2015; 64(48):1337-41.

50. Wheeler E, Jones TS, Gilbert MK, Davidson PJ. Centers for Disease Control and Prevention (CDC). Opioid overdose prevention programs providing naloxone to laypersons-United States, 2014. MMWR Morb Mortal Wkly Rep. 2015;64(23):631-5.

51. Price AM, Ilgen MA, Bohnert ASB. Prevalence and correlates of nonmedical use of prescription opioids in patients seen in a residential drug and alcohol treatment program. J Subst Abus Treat. 2011;41(2):208-14.

52. Bohnert ASB, Eisenberg A, Whiteside L, Price A, McCabe SE, Ilgen MA. Prescription opioid use among addictions treatment patients: nonmedical use for pain relief vs. other forms of nonmedical use. Addict Behav. 2013; 38(3):1776-81.

53. Bolck A, Croon M, Hagenaars J. Estimating latent structure models with categorical variables: one-step versus three-step estimators. Polit Anal. 2004; 12(1):3-27.

54. Clark S, Muthén B. Relating latent class analysis results to variables not included in the analysis. Los Angeles: University of California; 2009. [cited 2018 May 3]. Available from: https://www.statmodel.com/download/ relatinglca.pdf.

55. Vermunt J. Latent class modeling with covariates: two improved three-step approaches. Polit Anal. 2010;18(4):450-69. 